\title{
Photonic Chip Biosensor for Early-stage Cancer Diagnosis
}

\section{---- Design of silicon wire evanecence field "lab on a chip" biosensors}

\author{
Najla Najeeb ${ }^{* 1}$, Yaping Zhang ${ }^{2}$, Christopher Mellor ${ }^{3}$, Trevor Benson ${ }^{4}$ \\ 1,2Department of Electrical and Electronic Engineering, University of Nottingham Ningbo China, 199 Tainkang East \\ Road, Ningbo, 315100, China \\ 3School of Physics and Anstronomy, University of Nottingham, University Park, Nottingham NG7 2RD, UK \\ ${ }^{4}$ School of Electrical and Electronic Engineering, University of Nottingham, University Park, Nottingham NG7 2RD, \\ UK. \\ ${ }^{*}$ najla.najeeb@nottingham.edu.cn; ${ }^{2}$ yaping.zhang@nottingham.edu.cn; ${ }^{3}$ chris.mellor@nottingham.ac.uk; \\ ${ }^{4}$ trevor.benson@nottingham.ac.uk
}

\begin{abstract}
This paper introduces a general design of a silicon wire "lab on a chip" photonic biosensor for medical diagnosis application, especially for early-stage cancer diagnosis. The sensitivity of detection of the biosensors developed is at least 10-100 times more sensitive than that of current commercial biosensors.
\end{abstract}

\section{Keywords}

Silicon wire evanescent field biosensor; "Lab on a chip"; cancer early-stage diagnosis; sensitivity of detection; label-free detection; real time detection; multi-analytes' parallel detection.

\section{Introduction}

Reports from Xinhua Website (Xinhua News, 2015) indicates that the cancer cases in China accounts for $1 / 5$ of the total cancer cases globally, while the death cases due to cancer in China accounts for $1 / 4$ of the total death cases in the world. The lower survival rate in China compared with developed countries, such as USA, was mainly due to the late diagnosis of cancer cases. As the Chinese society is getting older, problems from environmental pollution and food security and so on has become serious, the above figures would get worse if no immediate effective measures are taken into action.

MicroRNAs (also miRNAs) have been identified as promising biomarkers for human diseases, including cancers (Calin et al, 2006; Waber et al., 2010). Many methods have been explored for the detection of miRNAs (Deng, et al., 2014; Degliangeli, et al., 2014; Roy, et al., 2011; Driskell, et al., 2008), but they are mostly too expensive and time consuming, requiring specialised laboratory equipments, and tedious incubation steps (Wen et al., 2012). Research has also been carried out with implementing silicon nanowire technology and some progresses has been made (Qavi, et al., 2011; Densmore, et al., 2009; Pandraud,et al., 2000; Ymeti, et al., 2005; Zhang, 2013). The development of "lab on a chip" photonic biosensors with high sensitivity of detection, capable of detecting disease-associated miRNAs, would be beneficial as an effective tool for the early-stage diagnosis of various disesases, especially cancers, as miRNAs are well preserved in various human specimens, including urine (Waber et al., 2010). We propose to design highly sensitive "lab on a chip" biosensors by incorprating many stand-alone Mach-Zehnder interferometer (MZI) biosensor elements with a multi-mode interferometer (MMI) optical coupler for multiple miRNA biomarks' detections. The complete "lab on a chip" biosensor is capable of detecting multiple miRNAs in clinical samples, such as urine, blood, rapidly and simultaneously in real-time without requiring labelling and any amplification processing.

\section{Configuration of "Lab on a chip" Biosensors}

A typical "lab on a chip" presented here is based on an UK patent of the author (Zhang, 2010). The single MZI sensing element is shown in Fig. 1; the multi-mode interferometer (MMI) optical coupler is shown in Fig. 2 and 3; the complete "lab on a chip" silicon wire photonic biosensor of our research is shown in Fig. 4, and the cross-section of the sensing window is shown in Fig. 5. 


\section{Single MZI sensing element}

A Mach-Zehnder Interferometer (MZI), served as a single sensing element, as shown in Fig. 1, is used to detect the phase change in the sensing arm due to the interaction of antibody (receptor) and the targeted molecules (shown in Fig. 5), with respect to a reference arm. Built-in reference arms are able to compensate for the variations, such as the refractive index fluctuations of both the waveguide materials and the bulk sample, non-specific absorption, and other unidentified causes, resulting from such variables as temperature fluctuations, mechanical changes and non-specific binding in the bulk sample.

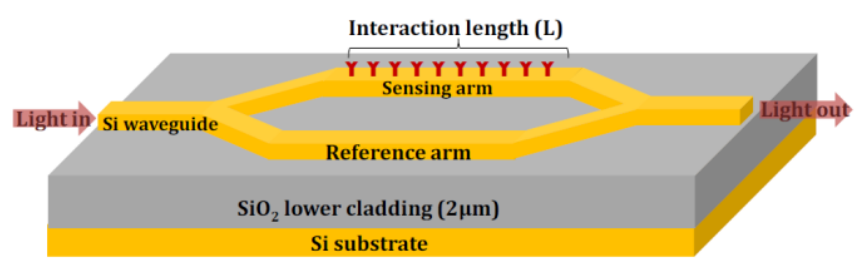

FIG. 1 ILLUSTRATION OF A MZI SENSING ELEMENT

The resulting phase difference at the output can be obtained from Eq. 1:

$$
\Phi_{S, R}=\frac{2 \pi}{\lambda} \cdot\left[N_{\text {eff } f_{s} s}-N_{\text {eff }, R}\right] \cdot L
$$

where the $N_{\text {eff, } S}$ and $N_{\text {eff, } R}$ are the effective refractive index of the sensing and reference arms respectively, and the $L$ is the interaction length of the receptor and analytes.

\section{An optical coupler of $1 \times N M M I$}

Fig. 2 shows the schematic diagram of a $1 \times 2 \mathrm{MMI}$ structure.

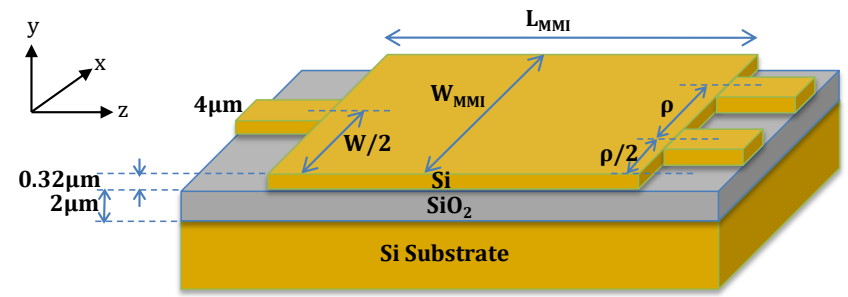

FIG. 2 SCHEMATIC DIAGRAM OF A 1X2 MMI STRUCTURE

Inside the MMI cavity the beam is split into numerous modes and the symmetric intensity pattern is repeated periodically at the intervals of $\Lambda$ along the guide where,

$$
\Lambda=\frac{n_{e f f} W_{M M I}^{2}}{\lambda}
$$

Here neff is the effective refractive index of the MMI cavity, $\mathrm{W}_{\mathrm{MMI}}$ the width of the MMI cavity and $\lambda$ is the operational wavelength. neff is the effective refractive index of the fundamental mode of the MMI cavity.

For a $1 \times \mathrm{N}$ MMI, the $\mathrm{N}$ images formed are equally spaced across the multimode guide. The distance between the images is known as the 'Pitch', $\rho=W_{M M I} / N$. The length of a $1 \times \mathrm{N}$ splitter (i.e., optical coupler) is found by $L_{m m i}=\Lambda / N$.

$$
L_{m m i}=\frac{\Lambda}{N}=\frac{n_{e f f} W^{2}}{N \lambda}
$$

In the presented work, the pitch is fixed at $15 \mu \mathrm{m}$ to prevent interference between the output waveguides. $W_{\text {MMI }}$ is determined by the number of output waveguides, $\mathrm{N}$, where $\mathrm{W}_{\mathrm{MMI}}=\mathrm{N} \rho$.

In our design, a $1 \times 20 \mathrm{MMI}$ optical coupler has been designed with the following parameters: waveguide width and height was kept at $4 \mu \mathrm{m}$ and $0.32 \mu \mathrm{m}$ respectively. Operating wavelength for all devices simulated is $1.55 \mu \mathrm{m}$. Using neff $=3.065$ and $\mathrm{W}_{\text {мm }}=300 \mu \mathrm{m}$ ( $\rho=15 \mu \mathrm{m}$ and $\left.\mathrm{N}=8\right)$ Lмmi can be calculated to be $8897 \mu \mathrm{m}$. A longer MMI length of $9300 \mu \mathrm{m}$ is simulated first to find the exact length at which the light converges into twenty equal powers.

Optimum cavity length is obtained when the power levels of all outputs are similar, at this optimum length the device will have its lowest insertion loss and highest uniformity as shown in Fig. 3.
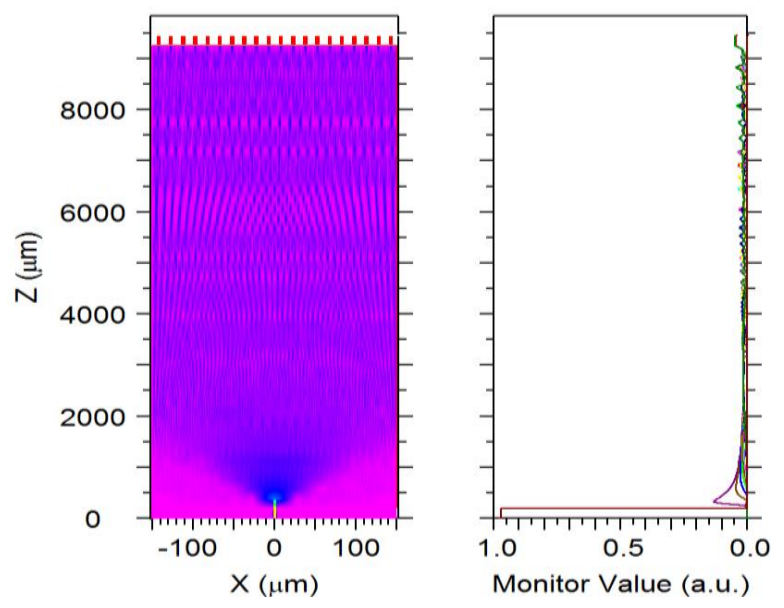

FIG. 3 SIMULATION AND DESIGN OF A 1X20 MMI STRUCTURE

\section{A complete "lab on a chip" biosensor}

Fig. 4 illustrates the configuration of our research of a complete "lab on a chip" photonics biosensor, which consists of an 1× N MMI optical coupler and N standalone MZI biosensing elements: 


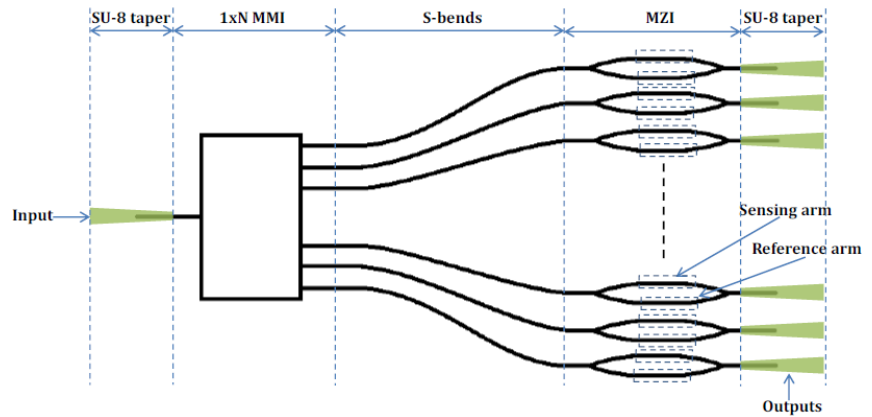

FIG. 4 ILLUSTRATION OF A COMPLETE 'LAB ON A CHIP' PHOTONIC BIOSENSOR

A MMI was employed to combine many stand-alone MZI biosensor elements to form a chip-level biosensor array, which enable real-time and label-free monitoring and parallel detection of various analytes in one or multiple test samples.

\section{Principle of the operation of the "Lab on a chip"}

At the sensing window an optical waveguide surface is functionalised with antibodies. In this work, highly sensitive silicon-on-insulator (SOI) waveguides are used (Fig. 5). Target molecules binding with the antibodies cause a phase change in the propagating light in the sensing arm that is proportional to the concentration of the target molecules.

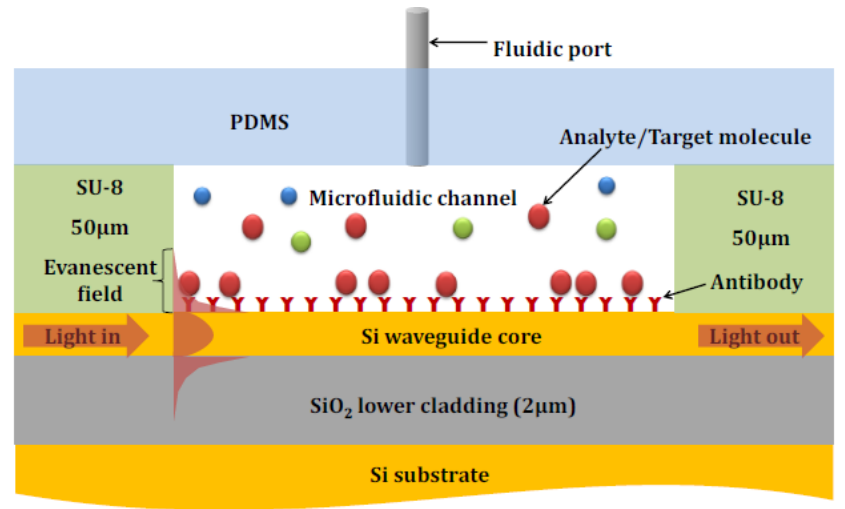

FIG. 5 ILLUSTRATION OF THE DETECTION PRINCIPLE: TARGET MOLECULES BINDS WITH THE ANTIBODIES COATED ON THE SENSING ARM WHICH INDUCES A PHASE CHANGE IN THE SENSING ARM COMPARED WITH THE REFERENCE ARM.

\section{Experimental Setup and Preliminary Results}

A $1 \times 20$ MMI optical coupler has been successfully fabricated and tested in the lab, as shown in the Fig. 6 with a lab set-up shown in Fig. 7. Fig. 6a shows successful operation of a fabricated 1x20 MMI optical coupler; Fig. $5 b$ and $5 c$ show the Environmental Scanning Electron Microscopy (ESEM) images of an
SU-8 taper and the Y-splitter of an MZI. The asymmetric Y-splitter is implemented here for the introduction of an initial phase difference to the sensing and reference arms, so as to have a better working point.

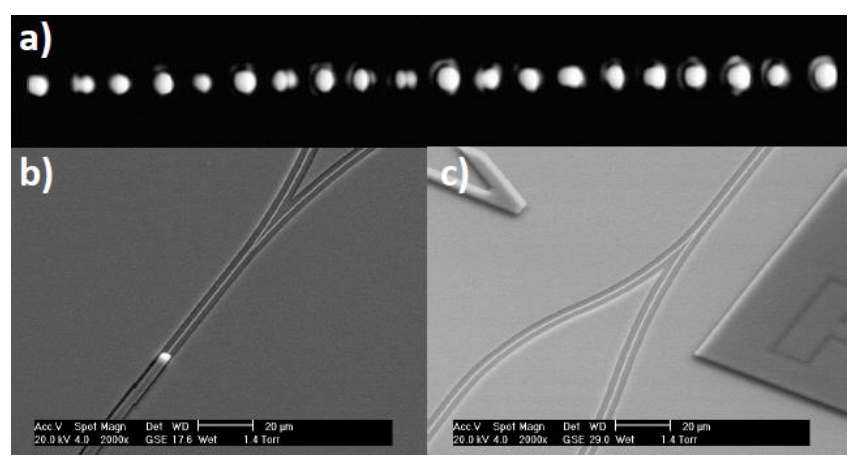

FIG. 6 a) OUTPUT OF AN 1×20 MMI OPTICAL COUPLER; b) ESEM IMAGE OF SU-8 INPUT TAPER AND Y-SPLITTER OF AN MZI; c) ESEM IMAGE OF A Y-SPLITTER OF AN MZI WITH ASYMEETRIC SENSING AND REFERENCE ARMS FOR BUILT-IN INITIAL PHASE SHIFT.
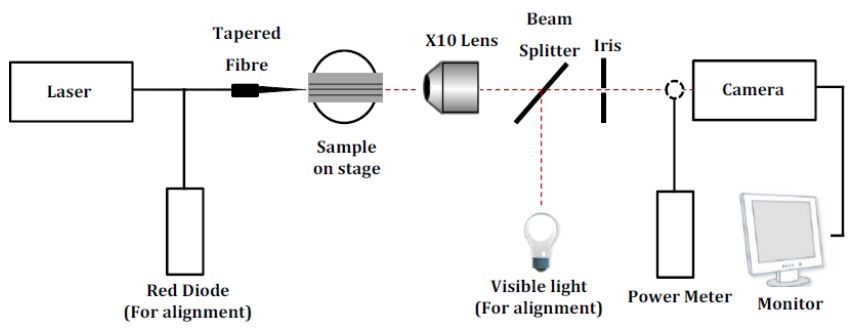

FIG. 7 BLOCK DIAGRAM OF OPTICAL BENCH SET-UP

Numerical calculations shows refractive index resolution of $2.7 \times 10^{-8}$ is possible for an MZI with sensing arm of length $15000 \mu \mathrm{m}$, which is 10 times more sensitive than reported MZI sensors (Zhang, 2010, Zhang, 2013).

\section{Conclusions}

The design of a complete "lab on a chip" silicon wire photonic biosensor, with a configuration of $1 \times 20 \mathrm{MMI}$ + 20 MZIs has been reported, and the optical lab test results have been provided. Simulation result of the sensitivity of detection for a predicted length of the sensing arm is 10 times more than previously reported MZI sensor. Further research and experiments on the miRNA biomarks' detections for the medical applications will be addressed.

\section{ACKNOWLEDGMENT}

This research activity has been funded by the University of Nottingham Ningbo China's Sandpit project and intercampus PhD scholarship. 


\section{REFERENCES}

Calin, G.A, Croce, C.M., 2006. Nat. Rev. Cancer 6, pp.857-866.

Deng, H., Shen, W., Ren, Y., Gao, Z., 2014. Biosensors and Bioelectronics, 54, 650-655.

Degliangeli, F., Kshirsagar, P., Brunetti, V., Pompa, P. P., Fiammengo, R., 2014, Journal of the American Chemical Society, 136, 2264-2267.

Densmore, A., Vachon, M., Xu, D.-X., Janz, S., Ma, R., Li, Y.H., Lopinski, G., Delâge, A., Lapointe, J., Luebbert, C.C., Liu, Q.Y., Cheben, P., and Schmid, Y.H., 2009, Optics Letters, 34 (23), 3598-3600.

Driskell, J. D., Seto, A. G., Jones, L. P., Jokela, S., Dluhy, R. A., Zhao, Y. P., Tripp, R. A., 2008, Biosensors and Bioelectronics, 24, 923-928.

Pandraud, G., Koster, T.M., Gui, C., Dijkstra, M., van der Berg, A., Lambeck, P.V., 2000, Sens. Actuators A 85, 158162.

Qavi, A. J., Kindt, J. T., Gleeson, M. A., Biley, R. C., 2011, Analytical Chemistry, 83, 5949-5956.

Roy, S., Soh, J. H., Gao, Z., 2011, Lab on a Chip, 2011, 11, 1886-1894.

Waber, J. A., Baxter, D. H., Zhang, S., Huang, D. Y., Huang, K. H., Lee, M. J., Galas, D. J., Wang, K., 2010, Clinical Chemistry, 56, 1733-1741.

Wen, Y., Per, H., Shen, Y., Xi, J., Lin, M., Lu, N., Shen, X., Li, J., Fan, C., 2012. Scientific Reports 2, pp.867.

Xinhua News: http://news.xinhuanet.com/politics/2015$\underline{02 / 03 / \mathrm{C} \quad 1114235376 . h t m}$

Ymeti, A., Kanger, J.S. , Greve, J. , Besselink, G.A.J., Lambeck, P.V., Wijn, R., Heideman, R.G., 2005, Biosensors and Bioelectronics, Vol. 20, pp.1417-1421.

Zhang, Yaping, 2013, Progress in Natural Science: Materials International 23, 481-486

Zhang, Yaping, 2010, GB2437543 B.

Najla Najeeb received her BEng (Hons) degree in Electronic and Communications from the University of Nottingham in 2011. She is currently pursuing her doctoral studies at the University of Nottingham Ningbo China. Her research involves the complete design, fabrication and testing of an Optical Biosensor device that will allow simultaneous and instantaneous label-free detection and identification of various analytes and molecular interaction in one or more test samples, with the help of microfluidics.
Yaping Zhang is the 2014 Zhejiang 1000-Talent. She obtained her BSc in Applied Optics in 1983 from Shandong University in China, MSc in Applied Physics in 1989 from the Defense University of Technology in China, and $\mathrm{PhD}$ in Optoelectronics in 2002 from The University of Nottingham in the UK. She has more than 30 years experience in the research of photonics and more than 10 years' recently industrial working experience in the UK and have successfully designed various most advanced commercial telecommunication optoelectronics products for global reknown companies.

Trevor M. Benson received a First Class honors degree in Physics and the Clark Prize in Experimental Physics from the University of Sheffield in 1979, a PhD in Electronic and Electrical Engineering from the same University in 1982 and the DSc degree from the University of Nottingham in 2005.

After spending over six years as a Lecturer at University College Cardiff, Professor Benson moved to The University of Nottingham in 1989. He was promoted to a Chair in Optoelectronics in 1996, having previously been Senior Lecturer (1989) and Reader (1994). Since October 2011 he has been Director of the George Green Institute for Electromagnetics Research at The University of Nottingham. Professor Benson's research interests include experimental and numerical studies of electromagnetic fields and waves with particular emphasis on the theory, modeling and simulation of optical waveguides, lasers and amplifiers, nano-scale photonic circuits and electromagnetic compatibility.

He is a Fellow of the Institute of Engineering Technology (FIET) and the Institute of Physics (FInst.P). He was elected a Fellow of the Royal Academy of Engineering in 2005 for his achievements in the development of versatile design software used to analyze propagation in optoelectronic waveguides and photonic integrated circuits.biography here.

Christopher J. Mellor received a First Class honors degree in Physics from the University of Birmingham in 1983, a PhD in Physics from the same University in 1986.

He is the Director of the Nanofabrication Facility, School of Physics and Astronomy, University of Nottingham. In this project Dr Mellor will design the fabrication processes and sequences to make the microfluidic chips with integrated photonic waveguides.

His current research is focussed on the area of quantum control which includes the low temperature study of nanoelectromechanical systems (NEMS), spintronic devices, the fabrication of ion chips and photonic integrated circuits for use in ultra-cold atom experiments. As well as low temperature and semiconductor physics experience, Dr Mellor also has expertise in the area of atomic force microscopy at both room and low temperature. 